\title{
ROLE OF CANINE FOSSA PUNCTURE IN ENDOSCOPIC SINUS SURGERY - OUR EXPERIENCE
}

\author{
J. S. Matta ${ }^{1}$,Nikhil Soni², S. S. Kaushik ${ }^{3}$ \\ 1 Professor and HOD, Department of ENT, American International Institute of Medical Sciences, Udaipur. \\ ${ }^{2}$ Senior Resident, Department of ENT, American International Institute of Medical Sciences, Udaipur. \\ ${ }^{3}$ Senior Resident, Department of ENT, American International Institute of Medical Sciences, Udaipur.
}

\begin{abstract}
\section{BACKGROUND}

Objectives- Chronic rhinosinusitis is a chronic disease that involves long-term inflammation of nasal and paranasal sinus mucosa (Benninger et al 2003). It is extremely a common condition affecting about $15 \%$ population. The maxillary sinus is one of commonest sinus to affect. Aetiology of chronic maxillary sinusitis is either rhinogenous or dental origin as proposed. The evidence of chronic maxillary sinusitis in the population was $0.02 \%$ and dental cause was found in $40.6 \%$ of cases. Nowadays, we perform endoscopic sinus surgery to eradicate the pathology and widening of ostiomeatal complex, but there are so many hidden area of maxillary sinus which cannot be reached by ESS alone. To overcome these shortcomings, canine fossa puncture with endoscopic sinus surgery is a useful procedure to fully visualise maxillary antrum and to deal with pathology in it. So we have conducted this study in our Institution to see the impact of Canine Fossa Puncture (CFP) with clearance of the maxillary sinus during endoscopic sinus surgery in severely diseased maxillary sinus.
\end{abstract}

Study Design - Retrospective.

\section{MATERIALS AND METHODS}

60 patients underwent canine fossa puncture with endoscopic middle meatal clearance of the maxillary sinus. At an average postoperative time of 3 months, all patients underwent post-op CT scan. Lund-Mackay scoring system was applied on pre- and post-op CT scans.(5)

\section{RESULTS}

72 sides were taken for analysis from 60 patients. All the patients were diagnosed with total opacification of maxillary sinus on contrast enhanced CT scan. On preoperative contrast enhanced computed tomography of nose and paranasal sinuses according to Lund-Mackay scoring system for all sinuses were scored $16.45 \pm 3.6$ and for maxillary sinus was 2 . Computed tomography of nose and paranasal sinuses taken at 3 months post-operatively according to Lund-Mackay scoring system for all sinuses were $5.45 \pm$ 0.98 and for maxillary sinus was $0.83 \pm 0.98$.

\section{CONCLUSION}

CFP is a useful technique to address the severely diseased maxillary sinus and is associated with significantly better symptom control compared with the transnasal middle meatal antrostomy clearance of the maxillary sinus.

\section{KEYWORDS}

CFP - Canine Fossa Puncture, MMA - Middle Meatus Antrostomy, ESS - Endoscopic Sinus Surgery.

HOW TO CITE THIS ARTICLE: Matta JS, Soni N, Kaushik SS. Role of canine fossa puncture in endoscopic sinus surgery - our experience. J. Evolution Med. Dent. Sci. 2017;6(1):23-26, DOI: 10.14260/Jemds/2017/7

\begin{abstract}
BACKGROUND
The maxillary sinus is the largest of the paranasal sinuses. It fills body of maxilla and is pyramidal in shape. The base is medial and forms much of the lateral wall of the nasal cavity. The floor, which often lies below the nasal floor, is formed by the alveolar process and part of palatine process of maxilla. It is related to the roots of the teeth, especially the 2 nd premolar and $1^{\text {st }}$ molar, but may extend posteriorly to the 3rd molar tooth or anteriorly to incorporate the $1^{\text {st }}$ premolar and sometimes the canine.
\end{abstract}

Financial or Other, Competing Interest: None.

Submission 12-12-2016, Peer Review 24-12-2016,

Acceptance 27-12-2016, Published 02-01-2017.

Corresponding Author:

Dr. J. S. Matta,

1 Sardarpura,

Udaipur-313001,

Rajasthan.

E-mail:drjsmatta@gmail.com

DOI: $10.14260 /$ jemds $/ 2017 / 7$
The roof of sinus forms the major part of the floor of the orbit. It contains the infra-orbital canal which may exhibit dehiscence. The lateral truncated apex of the pyramid extends into the zygomatic process of maxilla and may reach the zygomatic bone, in which case it forms the zygomatic recess which throws a $\mathrm{V}$-shaped shadow over the antrum on a lateral radiograph. The facial surface of maxilla forms its anterior wall and is grooved internally by a delicate canal (canalis sinuosus), which houses the anterior superior alveolar nerve and vessels as they pass forwards from the infra-orbital canal. The posterior wall is formed by the infratemporal surface of the maxilla: it contains alveolar canals that may produce ridges in the sinus and that also conduct the posterior superior alveolar vessels and nerves to the molar teeth. The medial wall is deficient postero-superiorly at the maxillary hiatus, a large opening which is partially closed in an articulated skull by portions of the perpendicular plate of palatine bone, the uncinate process of ethmoid bone, the inferior nasal concha, the lacrimal bone and the overlying nasal mucosa, to form an ostium and anterior and posterior fontanelles. The ostium usually opens into the inferior part of 
the ethmoidal infundibulum, and thence into the middle meatus via the hiatus semilunaris (the hiatus forms the area above the superior edge of the uncinate process). The fontanelles are covered only by periosteum and mucosa and may contain accessory ostia.

All openings are nearer the roof than the floor of the sinus, which means that the natural drainage of the maxillary sinus is reliant on an intact mucociliary escalator: the cilia of the sinus mucoperiosteum normally beat towards the ostium. The maxillary sinus may be incompletely divided by septa; complete septa are very rare. The thinness of its wall is clinically significant in determining the spread of tumours from the maxillary sinus. A tumour may push up the orbital floor and displace the eyeball; project into the nasal cavity causing nasal obstruction and bleeding; protrude onto the cheek causing swelling and numbness if the infraorbital nerve is damaged; spread back into the infratemporal fossa causing restriction of mouth opening due to pterygoid muscle damage and pain or spread down into the mouth, loosening teeth and malocclusion. Hypoplasia of maxillary antrum is present in up to $0.3 \%$ of the population.

\section{Ostiomeatal Complex}

The term ostiomeatal complex or ostiomeatal unit refers to the area that includes the maxillary sinus ostium, ethmoid infundibulum and the hiatus semilunaris. It is the common pathway for drainage of secretions from the maxillary and anterior group of ethmoidal sinuses, where the uncinate process attaches to the lateral nasal wall, the complex also drains the frontal sinus.

Endoscopic Sinus Surgery (ESS) is performed on patients with chronic rhinosinusitis with or without nasal polyposis who are refractory to medical therapy. The maxillary sinus is one of the most commonly affected sinus with other paranasal sinus (anterior ethmoids mainly) and endoscopic approach is mainly focused on widening of natural ostium (middle meatus antrostomy), this provides adequate access to the posterior, lateral and superior regions, but this technique is unsatisfactory for accessing anterior, medial, inferior areas of maxillary antrum. In such cases, many otolaryngologists occasionally choose the traditional Caldwell-Luc operation or inferior meatal antrostomy to visualise the blind area. However, these procedures provide limited access and associated with significant morbidities. To overcome these problems, canine fossa puncture with ESS is highly effective means of clearing disease from all these inaccessible area as well as visualisation of these areas to assess clearance of disease and with minimal complications as compared to traditional procedures. Studies show that the optimal placement of the CFP should be at the junction of imaginary mid pupillary line and a horizontal line drawn through the floor of the nasal vestibule. The said area of CFP decreases the risk of injury to major nerve trunks. Other than CRS, nasal polyposis, canine fossa puncture is useful in fungal sinusitis, eosinophilic mucin, mucopyoceles, foreign bodies, any dental pathology or any combination thereof.

The study is designed to assess the outcome of canine fossa puncture additional to ESS (MMA) in patients of nasal polyposis and CRS.
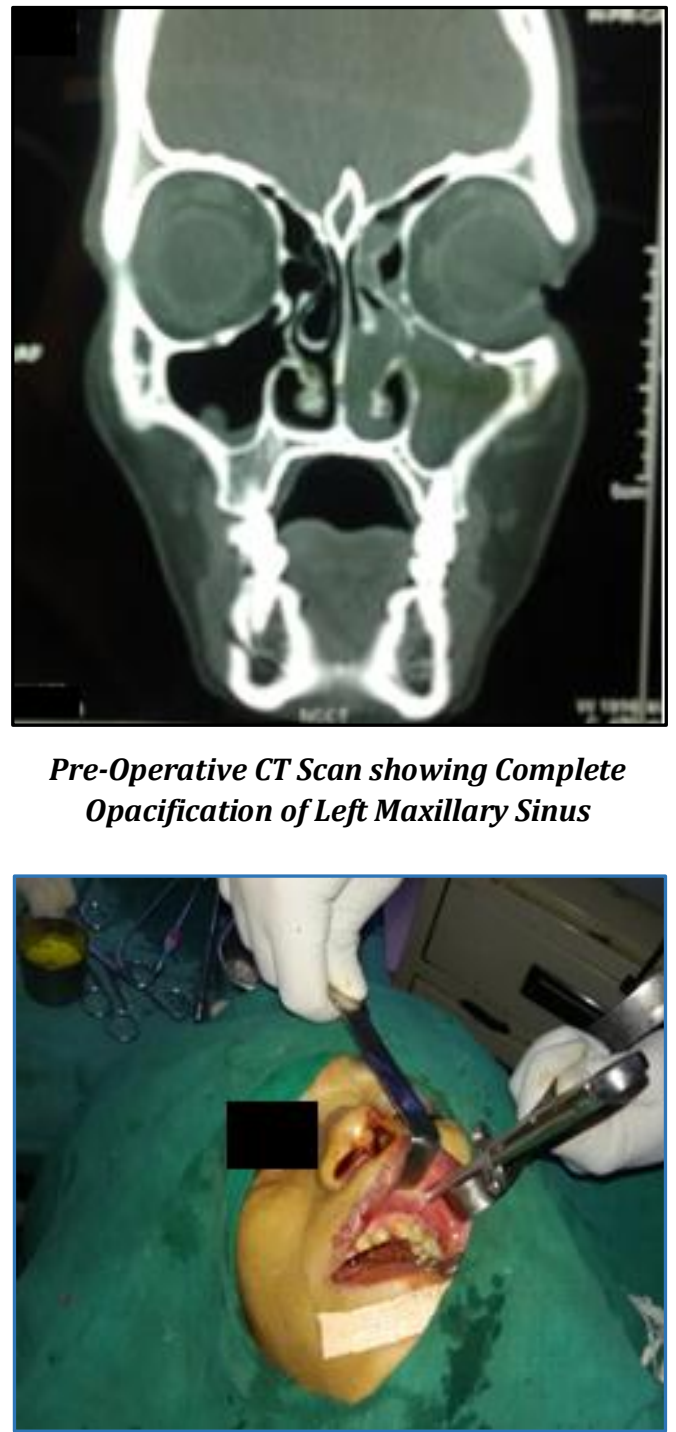

Figure showing Site of Canine Fossa Puncture

\section{MATERIALS AND METHODS}

This is a retrospective study performed in patients suffering from chronic rhinosinusitis with or without nasal polyposis with extensive disease in maxillary sinus (total or near total opacification) seen on preoperative CT scans. These patients underwent endoscopic sinus surgery with canine fossa puncture between December 2015 and July 2016 at our Institute. The exclusion criteria were as follows: patients younger than 12 years, because before that maxillary sinus are not fully developed. Patient who had undergone CaldwellLuc operation and patient suffering from acute sinus infection. Patient who declined for the procedure or opted out of the study. All the patients went through a preoperative contrast enhanced computed tomography of nose and paranasal sinuses. All the routine clinical laboratory investigations were done preoperatively. All the patients were fit for general anaesthesia. All the surgical procedures were done under general anaesthesia. Before giving general anaesthesia, nasal decongestion was achieved by using $4 \%$ lignocaine with adrenaline solution. The techniques used for endoscopic sinus surgery were those done conventionally and extent of surgery was determined according to the involvement of sinuses, additional to this technique canine fossa puncture was done on the side where there was 
extensive disease of maxillary sinus which could not be cleared through endoscopic widening of natural ostium.

The entry point of maxillary sinus trocar in the canine fossa puncture was the intersection of the mid pupillary line and a horizontal line running along the lower border of the nasal alae as recommended by Wormald PJ et al.(1) A $4 \mathrm{~mm}$ opening was created, which allowed insertion of a curved 4 $\mathrm{mm}$ microdebrider blade (Stryker instruments). The antrum was visualised with rigid $30^{\circ}, 70^{\circ}$ endoscope and the pathological mucosa was removed with microdebrider inserted in canine fossa opening.

The nasal Merocel was placed in the nasal cavity soaked in paraffin and antibiotic ointment, which was removed 2 days postoperatively and the patients were discharged the following day. After surgery, we prescribed a dose of $30 \mathrm{mg}$ prednisolone tablet for 6 days in three divided doses followed by tapering doses of $20 \mathrm{mg}$ for another 4 days with antibiotics. The patients were educated regarding the method of saline irrigation. Each patient visited our OPD once a week for one month twice a month thereafter. On each visit meticulous endoscopy was done and changes were noted and complications were noted and taken care off.

\section{Lund-Mackay CT Scoring System}

\begin{tabular}{|c|c|c|}
\hline Sinuses & Right & Left \\
\hline Maxillary sinus & & \\
\hline Anterior ethmoidal & & \\
\hline Posterior ethmoid & & \\
\hline Frontal & & \\
\hline Sphenoid & & \\
\hline Ostiomeatal complex & & \\
\hline Total & & \\
\hline
\end{tabular}

Sinuses: 0 = No Opacifications, 1 = Partial Opacification, 2 $=$ Total Opacification.

Ostiomeatal Complex: $0=$ Not Obstructed, $2=$ Obstructed Note: Lund-Mackay CT scoring system was used in this study.(2)

\section{RESULTS}

In our study, sixty patients were included. All the patients had chronic rhinosinusitis refractory to medical therapy out of which 72 sides underwent canine fossa puncture. Out of 60 patients, 42 were male and 18 were female. The average age was 32.5 (16 - 60 yrs.).

In our study, most common symptom in patient with CRS was nasal discharge (100\%), nasal obstruction (80\%), headache $(10 \%)$ and postnasal drip (10\%).

On preoperative contrast enhanced computed tomography of nose and paranasal sinuses according to Lund-Mackay scoring system for all sinuses were $16.45+3.6$ and for maxillary sinus was 2 .

Computed tomography of nose and paranasal sinuses taken at 3 months post-operatively according to LundMackay scoring system for all sinuses were $5.45+0.98$ and for maxillary sinus was $0.83+0.98$.

In our study, most common symptom in patient with CRS postoperatively was nasal obstruction (10\%), post nasal drip (4\%) and nasal discharge (2\%).

None of the patients had any type of complication immediate postoperative, but one patient complains of facial numbness at 3-month interval.

\section{DISCUSSION}

Canine fossa puncture with ESS is an invaluable technique for better and complete visualisation of hidden part of maxillary sinus, which cannot be seen by ESS. It has been recommended as a procedure of choice for complete clearance of maxillary sinus mucosal disease by Sathananthar et al.(3) This is used in many conditions of sinus such as CRS with or without nasal polyposis, AFRS, inverted papilloma, eosinophilic mucin, fungal sinusitis, chronic invasive aspergillosis, mucocoele and maxillary cyst. It is clear that it offers technical superiority in access to the anterior and inferior portions of maxillary sinus. As our study demonstrates that canine fossa puncture with ESS is very helpful in clearing disease in severely disease maxillary sinus. According to Lund-Mackay scoring system, score for maxillary sinus was 2 preoperatively and $0.83+0.98$ postoperatively. Interestingly, Lee et al noted no significant difference in outcome for patients with chronic rhinosinusitis and nasal polyposis treated with CFT veruses MMA without CFT. $(4,5)$ There have been suggestions that massive occlusive polyps in the maxillary sinus may resolve if the maxillary antrum is opened and aerated. However, there are no published long-term studies that have confirmed this suggestion. That is why there is different opinion on this technique.

However, many otorhinolaryngologist hesitate to adopt this technique for the fear of its potential complications.(6) The complications of the CFP are cheek swelling, facial pain, facial numbness, cheek pain, dental numbness, gingival complications and facial tingling which are the result of injury to branches of the infraorbital nerve as illustrated by Robinson et al.(7) To decrease the risk of complications, the site of puncture in canine fossa is an intersection between the mid-pupillary line and a line through the floor of the nasal vestibule as advocated as landmark by Wormald PJ et al. In our study, we advocate the same site of puncture as described by Wormald P J et al.(8) Only one patient complains of facial numbness, immediate post-operative period which resolve at third-month interval.

\section{CONCLUSION}

This study advocates the use of canine fossa puncture with ESS in cases of chronic rhinosinusitis with or without nasal polyposis and found out that this is really helpful in clearing disease from in access areas of maxillary sinuses such as anterior and inferior portions of sinus with minimal complications.

\section{REFERENCES}

[1] Tewfik MA, Wormald PJ. Planning for the canine fossa trephination approach. Operative Techniques in Otolaryngology Head and Neck Surgery 2010;21(2):1504.

[2] Lund VJ, Mackay IS. Staging in rhinosinusitis. Rhinology 1993;31(4):183-4.

[3] Sathananthar S, Nagaonkar S, Paleri V, et al. Canine Fossa puncture and clearance of the maxillary sinus for the severely diseased maxillary sinus. Laryngoscope 2005;115(6):1026-9. 
[4] Lee JY, Lee SH, Hong HS, et al. Is the canine fossa puncture approach really necessary for the severely diseased maxillary sinus during endoscopic sinus surgery? Laryngoscope 2008;118(6):1082-7.

[5] Byun JY, Lee JY. Canine fossa puncture for severe maxillary disease in unilateral chronic sinusitis with nasal polyp. Laryngoscope 2013;123(12):E79-84.

[6] Anand V, Santosh S, Aishwarya A. Canine fossa approaches in endoscopic sinus surgery - our experience. Indian J Otolaryngol Head Neck Surg 2008;60:214-17.
[7] Robinson SR, Baird R, Le T, et al. The incidence of complications after canine fossa puncture performed during endoscopic sinus surgery. Am J Rhinol 2005;19(2):203-6.

[8] Robinson S, Wormald PJ. Patterns of innervation of the anterior maxilla: a cadaver study with relevance to canine fossa puncture of the maxillary sinus. Laryngoscope 2005; 115(10):1785-8. 